\title{
RELACIÓN DEL REFLUJO GASTROESOFÁGICO Y MANIFESTACIONES RESPIRATORIAS, DESDE EL PUNTO DE VISTA DE LA GASTROENTEROLOGÍA PEDIÁTRICA

\section{Dra. M. Francisca Jaime M.}

Unidad de Gastroenterología Infantil, Departamento de Pediatría, Clínica Alemana de Santiago. Facultad de Medicina Universidad del Desarrollo. Departamento de Gastroenterología y Nutrición Pediátrica, Red de Salud UC-Christus.

\begin{abstract}
Gastroesophageal reflux is a frequent condition in the daily life of infants and older children. When reflux causes symptoms, it is called gastroesophageal reflux disease. Different extraesophageal symptoms have been frequently attributed to gastroesophageal reflux, however, new diagnostic techniques available, such as pHmetry with impedance measurement, have allowed us to evaluate and eventually dismiss such relationships. In this article we review the relationship between gastroesophageal reflux and laryngeal pathology, chronic cough, asthma and aspiration. In general terms, the empirical treatment of a presumed reflux is not recommended in asymptomatic patients, in whom its presence is not demonstrated by techniques such as pHmetry with impedance, given that therapeutic response is low and similar to placebo, with potential adverse effects.
\end{abstract}

Keywords: variable, measurement, definition, operationalize.

RESUMEN

El reflujo gastroesofágico es una condición frecuente en la vida diaria de lactantes y niños mayores. Cuando produce síntomas, se denomina enfermedad por reflujo gastroesofágico. Se ha atribuido frecuentemente diferentes síntomas extraesofágicos al reflujo, sin embargo, nuevas técnicas diagnósticas disponibles, como la pHmetría con medición de impedanciometría, han permitido evaluar y eventualmente descartar tales asociaciones. En este artículo se revisa la relación entre el reflujo gastroesofágico y patología laríngea, tos crónica, asma y aspiración. En términos generales, no se recomienda el tratamiento empírico de un supuesto reflujo en pacientes asintomáticos, en quienes tampoco esté demostrada su presencia por técnicas como la pHmetría con impedanciometría, dado que la respuesta terapéutica es baja y similar a placebo, con potenciales efectos adversos.

Palabras claves: reflujo gastroesofágico, aspiración, asma, niños.

\section{INTRODUCCIÓN}

El reflujo gastroesofágico (RGE) se define como el paso retrógrado de contenidos desde el estómago hacia el esófago. Es un fenómeno que ocurre diariamente incluso en personas sanas, sin embargo cuando produce síntomas molestos o consecuencias para la salud, se denomina enfermedad por reflujo gastroesofágico (ERGE) (1). Un metanálisis reciente, muestra que la ERGE puede presentarse en alrededor del $19 \%$ de niños mayores de 10 años (2).

En lactantes, el RGE es un fenómeno habitual, afectando alrededor de un 25\% de los menores de 6 meses y mucho más si se considera solo un episodio de regurgitación diario (2). Esto disminuye con el tiempo y en la mayoría de los casos no constituye enfermedad, definido dentro de los criterios de ROMA IV como regurgitaciones del lactante (3).

\section{Correspondencia:}

Dra. Francisca Jaime M.

Unidad de Gastroenterología Infantil, Clínica Alemana de Santiago

Av. Vitacura 5951, 3er piso, Vitacura - Santiago

Teléfono +56975174282

mjaime@alemana.cl
La ERGE puede manifestarse a nivel digestivo por Ios síntomas clásicamente asociados: pirosis y regurgitación. Ocasionalmente puede producir disfagia, dolor retroesternal e incluso hematemesis. En niños pequeños que no verbalizan, puede manifestarse como rechazo alimentario, llanto posterior a los episodios de reflujo, posturas anómalas de hiperextensión cervical, entre otros. Además puede producir lesiones erosivas en esófago secundarias a exposición excesiva al ácido, pero también a otros componentes como bilis y pepsina, llegando incluso a la estenosis esofágica y esófago de Barrett.

Actualmente se reconocen nuevas entidades respecto a la sintomatología esofágica, donde existe un continuo entre el daño causado por reflujo, y fenómenos de hipersensibilidad visceral: la esofagitis erosiva, la enfermedad por reflujo no erosivo, la hipersensibilidad al reflujo y la pirosis funcional, cuyas características se describen en la tabla 1 (4).

Diferentes síntomas y signos extraesofágicos se han relacionado con reflujo. En este artículo, se revisarán las manifestaciones respiratorias asociadas al reflujo gastroesofágico, la evidencia disponible sobre su asociación y tratamiento. 
Tabla 1. Características de las diferentes entidades asociadas a reflujo gastroesofágico y síntomas. ERGE: enfermedad por reflujo gastroesofágico.

\begin{tabular}{|c|c|c|c|c|}
\hline $\begin{array}{c}\text { Exposición esofágica al } \\
\text { reflujo en niveles patológicos }\end{array}$ & $\begin{array}{c}\text { Esofagitis } \\
\text { erosiva }\end{array}$ & $\begin{array}{c}\text { ERGE no } \\
\text { erosiva }\end{array}$ & $\begin{array}{c}\text { Hipersensibilidad } \\
\text { al reflujo }\end{array}$ & No \\
\hline $\begin{array}{c}\text { Síntomas asociados a } \\
\text { episodios de reflujo }\end{array}$ & Sí o No & Sí o No & Sí & No \\
\hline $\begin{array}{c}\text { Erosiones esofágicas en } \\
\text { endoscopía }\end{array}$ & Sí & No & No & No \\
\hline
\end{tabular}

\section{MANIFESTACIONES RESPIRATORIAS DEL REFLUJO GASTROESOFÁGICO}

En esta revisión se abordará los síntomas y signos laríngeos, la tos crónica, el asma y la aspiración. Iniciaremos con una descripción de los estudios diagnósticos que habitualmente se utilizan en pacientes con ERGE, y que han permitido clarificar el rol del reflujo en estas manifestaciones.

\section{Métodos diagnósticos de reflujo gastroesofágico}

No hay un solo estudio o estándar de oro que permita diagnosticar la ERGE, sino que la estrategia diagnóstica debe adaptarse a las características únicas de cada paciente.

Para el estudio del RGE inicialmente se utilizó la medición de pHmetría esofágica, en la que a través de una sonda con sensor de $\mathrm{pH}$, introducida a través de la nariz y con su extremo distal en esófago (o estómago, según las mediciones de interés), se mide el paso retrógrado de contenido ácido. Posteriormente se agregó la medición de impedanciometría intraluminal multicanal (IIM), que permite evaluar adicionalmente el paso de contenido líquido, gaseoso o mixto, sin importar su pH (figura 1). A partir de esto se ha clasificado el contenido del reflujo en ácido y no-ácido (que incluye al reflujo débilmente ácido y alcalino). Esto es importante para algunos grupos etarios como los lactantes, en quienes una gran parte de los síntomas se producen por reflujo no ácido, que no podría ser detectado por la pHmetría convencional. La principal utilidad de estos estudios es la correlación con síntomas, como se describirá más adelante. Las indicaciones actualmente aceptadas de $\mathrm{pH}-\mathrm{IIM}$ se describen en la tabla 2.

La endoscopía digestiva alta permite la visualización directa del daño causado en la mucosa por reflujo, sin embargo puede existir la ERGE sin tener lesiones mucosales. Permite además evaluar signos sugerentes de otras patologías, como estenosis esofágica y acalasia, y también obtener muestras para biopsia, importantes para el diagnóstico diferencial con esofagitis eosinofílica, patología que por lo demás se presenta con mayor frecuencia en pacientes con antecedentes de atopía y asma (Tabla 2). Se considera que la endoscopía digestiva tiene una baja sensibilidad, pero alta especificidad para el diagnóstico de reflujo (5).
Figura 1. Episodio de reflujo en pH-IIM (pHmetría con impedanciometría intraluminal multicanal). Se observa caída de las curvas de impedancia en todos los canales de medición. Las flechas muestran la dirección del contenido esofágico, en este caso, de distal hacia proximal (reflujo) y luego de proximal a distal (aclarado del reflujo). En este caso, se trata de un episodio de reflujo no ácido.

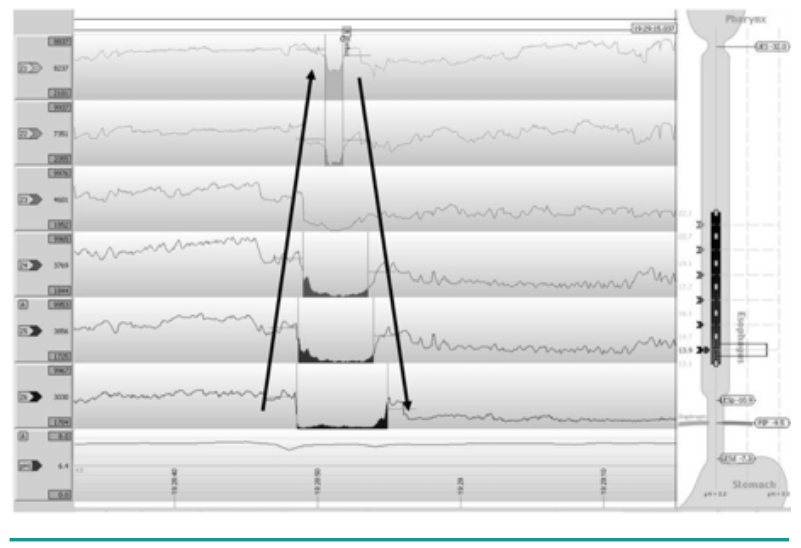

Otro estudio habitualmente utilizado son las imágenes radiológicas con uso de contraste, como la radiografía esófago-estómago-duodeno o el esofagograma. Estos estudios permiten detectar alteraciones anatómicas que pueden predisponer a reflujo gastroesofágico (hernia hiatal, estenosis esofágica, malrotación intestinal, estenosis pilórica, membrana duodenal, compresión extrínseca, etc). Si bien su informe habitualmente incluye la descripción de reflujo cuando se presenta durante el examen, no debería ser utilizado para el diagnóstico de ERGE (6), dado su baja sensibilidad y especificidad (51 y 58\%, respectivamente en un estudio pediátrico) (7). Además, expone a radiación al paciente y la tasa de hallazgo de malformaciones detectadas es baja, por lo que no se recomienda de rutina en la evaluación de pacientes con reflujo, pero sí cuando la sospecha de alteraciones anatómicas es alta.

El cintigrama o gammagrafía de vaciamiento gástrico se utiliza en pacientes con sospecha de gastroparesia, por ejemplo, pacientes con vómitos postprandiales tardíos, 
Tabla 2. Indicaciones para realizar pHmetría-IIM (impedanciometría intraluminal multicanal) y endoscopía digestiva alta. BRUE: evento breve resuelto inexplicado.

\section{Indicaciones para realizar $\mathrm{pH}-\mathrm{IIM}$}

1. Diferenciar pacientes con esofagitis no erosiva, hipersensibilidad esofágica y pirosis funcional.

2. Determinar la eficacia de la terapia de supresión ácida.

3. Evaluar la correlación de síntomas extra-esofágicos con reflujo ácido o no ácido.

- Síntomas respiratorios

- BRUE

- Síntomas respiratorios que no responden a terapia

- Otros síntomas extra-esofágicos

- Posturas tipo Sandifer

- Llanto inexplicado

- Erosión del esmalte dental asociado con enfermedades neurológicas

- Inflamación laríngea inexplicada

Indicaciones para realizar endoscopía digestiva alta (relacionadas a RGE)

1. Presencia de síntomas de alarma

2. Detectar complicaciones de reflujo: estenosis, esófago de Barrett

3. Detectar condiciones que predisponen a reflujo (por ej. hernia hiatal)

4. Detectar condiciones que se presentan similar a reflujo (por ej. esofagitis eosinofílica, esofagitis infecciosal

quienes podrían tener mayor predisposición al reflujo. Una adaptación de este examen permite evaluar episodios de paso de contenido gástrico a la vía aérea en pacientes con sospecha de aspiración. El examen consiste en la administración de alimentos (leche o agua) con trazadores como 99mTc; luego se detecta su actividad por gammacámaras, en un período de tiempo de alrededor de 120 minutos. Sin embargo, la técnica no está bien estandarizada en los diferentes centros, y no existen valores de normalidad para niños; existen pocos estudios pediátricos y la técnica tiene una capacidad limitada para detección de los episodios de aspiración (8).

Los niveles de pepsina en secreción traqueal o saliva se han utilizado igualmente para la evaluación de pacientes con manifestaciones respiratorias atribuidas a reflujo o aspiración, sin embargo existe producción de pepsina no sólo en el estómago, sino que en otros órganos, incluyendo pulmones. Un estudio reciente con pepsina salival mostró niveles elevados en lactantes sanos (9). La isoforma A de la pepsina podría ser útil por su mayor especificidad para el estómago, sin embargo aún falta mayor desarrollo de la técnica y de estudios que la evalúen (10).

El porcentaje de macrófagos cargados con lípidos del lavado broncoalveolar, no ha mostrado utilidad y ya no se utiliza para la evaluación de aspiración $(1,11)$.

\section{ERGE y BRUE en lactantes}

El evento breve resuelto inexplicado (BRUE, por su sigla en inglés) se atribuye a ERGE frecuentemente. En la gran mayoría de los casos la atribución se hace por clínica y no basado en estudios o evaluación por gastroenterólogo (12). En lactantes evaluados por $\mathrm{pH}-\mathrm{IIM}$, se ha evidenciado que hasta en $85 \%$ de los casos existe reflujo en niveles patológicos, pero solo en $11-42 \%$ de los casos, existe además una relación temporal y eventualmente causal entre reflujo y BRUE/apnea. Estos episodios son mayoritariamente no ácidos, por lo que la medición sólo de pHmetría es insuficiente en su estudio (13).

\section{ERGE y manifestaciones laríngeas}

Existe una serie de hallazgos en el examen laringeo que se han atribuido a reflujo (denominado reflujo faringolaríngeo); algunos de ellos se pueden encontrar frecuentemente en adultos sanos (14). Dado lo anterior, se ha desarrollado un escala de hallazgos de reflujo (o Reflux Finding Score, RFS), basado en la evaluación de 8 items relacionados con inflamación laringea: edema subglótico, obliteración ventricular, eritema/hiperemia, hipertrofia de la comisura posterior, tejido granulatorio y moco endolaringeo espeso, con diferentes puntos de corte para mejorar la sensibilidad y especifidad respecto al diagnóstico de reflujo (15). Sin embargo, un estudio pediátrico realizado en un centro de referencia mundial de patología aerodigestiva, mostró una deficiente correlación interobservador del RFS, así como también bajo rendimiento como estudio diagnóstico al comparar con reflujo diagnosticado por pHIIM, con sensibilidad de 95\% pero especificidad de $9 \%$ al considerar un punto de corte $>7$ del score, 0 una sensibilidad inaceptablemente baja de $14 \%$ y especificidad de $95 \%$ con punto de corte $>16$ (16). Por otro lado, los pacientes adultos con hallazgos en la nasofibroscopía sugerentes de reflujo tienen una baja respuesta a tratamiento con inhibidor de bomba de protones (IBP), cercana al $40 \%$ y similar a la 
respuesta al placebo (17). Por lo anterior, se sugiere que el diagnóstico de reflujo en niños no debe basarse solo en los hallazgos de la nasofibroscopía, evitando así el uso prolongado e innecesario de IBP, con los potenciales efectos secundarios que aún están describiéndose en la literatura (18).

\section{ERGE y tos crónica}

Por mucho tiempo se ha atribuido la tos crónica sin una causa evidente a la presencia de ERGE. Desde el punto de vista de la plausibilidad biológica, tanto la vía aérea como el esófago tienen un origen embrionario común, y comparten vías de inervación a través del vago. Dado lo anterior, el ascenso de contenido gástrico puede estimular terminales nerviosas hacia proximal, y secundariamente inducir reflejo de tos, sin que necesariamente el reflujo esté alcanzando la vía aérea. En adultos con tos crónica, se ha evidenciado incluso que la falta de respuesta a IBP se asocia más bien con un bajo umbral de sensibilidad para desencadenar reflejo de tos. Por otro lado, un paciente con tos crónica presenta frecuentemente episodios de presión abdominal positiva, con ascenso de contenido gástrico secundario.

Para evaluar la relación de tos con reflujo, es útil la medición de pHmetría-IIM. En esta se busca una relación temporal de los episodios de reflujo con la tos, a través de métodos estadísticos, sin embargo su principal problema es la falla en reportar cada episodio de tos (hasta un 90\%) (19), tanto por el mismo paciente en el caso de niños mayores, como por los cuidadores. Es por esto que se ha agregado otros métodos para detectar los episodios de tos, como la medición de presión gástrica (manometría) o la grabación de sonido, los que están muy poco disponibles en la práctica clínica diaria. A través de estudios que incluyen estos últimos métodos, se ha visto que dentro de los niños con tos crónica no explicada, alrededor de $40 \%$ tienen ERGE, tanto ácido como no ácido (13).

Una guía clínica basada en evidencia publicada recientemente, sugiere NO tratar empíricamente la ERGE en pacientes pediátricos con tos crónica si no tienen síntomas, signos o estudio compatible con esta patología (endoscopía digestiva alta, pH-IIM) (20), dado la baja efectividad del tratamiento y la potencialidad de efectos adversos asociados. En pacientes que sí tienen síntomas, signos 0 estudio compatible con ERGE, se sugiere tratamiento de acuerdo a las guías actuales de ERGE (1) y reevaluar la respuesta a terapia luego de 4-8 semanas.

\section{ERGE y asma}

El reflujo y el asma son dos fenómenos que pueden ocurrir frecuentemente, y por lo mismo pueden coexistir en un mismo paciente. Se ha descrito alta frecuencia (hasta $80 \%$, pero en general alrededor de 40-50\%) de reflujo en pacientes asmáticos, a través de estudios de pHmetría (21). Existen dos teorías que explicarían esta mayor frecuencia: La teoría del reflujo, en que el material refluido llegaría a la vía aerea en forma de microaspiración, y la teoría del reflejo, en que de forma similar a lo ya descrito en el párrafo anterior, dado el origen embronario común, el reflujo proximal estimularía receptores de vías vagales, con efectores a nivel bronquial, causando broncoconstricción (5). Por otro lado, la obesidad es un factor de riesgo común, importante y frecuentemente presente en pacientes con asma y reflujo. Factores físicos como la mayor presión negativa ejercida sobre el esófago durante la inspiración en pacientes con asma mal controlada, y la presión positiva sobre el abdomen durante la espiración y la tos, promueven el paso del contenido gástrico en forma retrógrada. Además el uso de medicamentos para el tratamiento del asma afecta el efecto antirreflujo de la unión gastroesofágica.

A pesar de lo descrito, el tratamiento con IBP no ha sido útil en mejorar el control del asma en niños sin evidencia de reflujo, por lo que su uso empírico en este último grupo de pacientes no se recomienda (1).

\section{ERGE y aspiración}

En un paciente con alteración de los mecanismos protectores de aspiración (por ejemplo, pacientes con patología neurológica, parálisis de cuerdas vocales, etc), el reflujo puede ser un factor que agrave los episodios respiratorios. Sin embargo, en estos pacientes puede haber no solamente RGE, sino también trastornos de motilidad esofágica que impidan el paso descendente del contenido deglutido, entre ellos, acalasia esofágica o disfunción cricofaríngea, evaluable a través de manometría esofágica, en que se produce retención faringea y posterior paso a vía aérea del material deglutido. Otras alteraciones de la motilidad esofágica como la acalasia esofágica o la atresia esofágica operada, pueden dificultar la llegada del bolo alimentario al estómago, o promover la permanencia de los alimentos a nivel esofágico y faringeo (22). En algunos de estos casos, el tratamiento del reflujo a través del uso de IBP no disminuye el riesgo de neumonía aspirativa, dado que persiste el riesgo de aspiración del contenido oral, y se ha asociado -con evidencia aún no concluyente- con mayor frecuencia a infecciones respiratorias.

\section{TRATAMIENTO}

El tratamiento de la ERGE depende de cada paciente y de las características del reflujo. El reflujo ácido podría beneficiarse del uso IBP. Sin embargo, su uso ha demostrado que principalmente cambia el $\mathrm{pH}$ del contenido refluido de ácido a no ácido, sin necesariamente disminuir su volumen o frecuencia (23). Esta consideración es importante particularmente en el caso de la ERGE en los lactantes y recién nacidos, donde el uso de IBP no ha mostrado utilidad en reducir los episodios de reflujo. La principal utilidad es la mejoría de la esofagitis por reflujo. Las guías actuales existentes de la Sociedad Norteamericana y Europea de Gastroenterología, Hepatología y Nutricion Pediátrica(1), recomiendan el uso de IBP (omeprazol, esomeprazol, lansoprazol) o de antagonistas del receptor histaminérgico tipo 2 (como ranitidina y famotidina) en pacientes con síntomas típicos de reflujo 0 exámenes que apoyen el diagnóstico (como la pHmetría-IIM). Como ya se comentó más arriba, el uso de IBP tiene una utilidad muy limitada 
en las manifestaciones extraesofágicas como la tos crónica y el asma mal controlada, con una respuesta muy similar a placebo.

\section{CONCLUSIONES}

La enfermedad por reflujo gastroesofágico es una patología frecuente en la población pediátrica, que ocasionalmente puede tener manifestaciones extra digestivas. Frecuentemente los síntomas respiratorios y particularmente los laringeos son sobre-atribuidos a ERGE, y los resultados del tratamiento actual con IBP no son óptimos.

No se recomienda el tratamiento empírico de reflujo en pacientes con síntomas extraesofágicos atribuidos a esta patología, sin evidencia clínica o de exámenes de ERGE.

\section{Conflictos de interés}

Declaro no tener conflictos de interés.

\section{REFERENCIAS}

1. Rosen $R$, Vandenplas $Y$, Singendonk $M$, Cabana M, DiLorenzo C, Gottrand F, et al. Pediatric Gastroesophageal Reflux Clinical Practice Guidelines: Joint Recommendations of the North American Society for Pediatric Gastroenterology, Hepatology, and Nutrition and the European Society for Pediatric Gastroenterology, Hepatology, and Nutrition. J Pediatr Gastroenterol Nutr. 2018;66(3):516-54.

2. Singendonk $M$, Goudswaard $E$, Langendam $M$, van Wijk $M$, van Etten-Jamaludin F, Benninga $M$, et al. Prevalence of Gastroesophageal Reflux Disease Symptoms in Infants and Children. Journal of Pediatric Gastroenterology and Nutrition. 2019;68(6):811-7.

3. Benninga MA, Faure C, Hyman PE, St James Roberts I, Schechter NL, Nurko S. Childhood Functional Gastrointestinal Disorders: Neonate/Toddler. Gastroenterology. 2016;130:1519-26.

4. Aziz Q, Fass R, Gyawali CP, Miwa H, Pandolfino JE, Zerbib F. Functional Esophageal Disorders. Gastroenterology. 2016;150:1369-79.

5. Broers C, Tack J, Pauwels A. Review article: gastrooesophageal reflux disease in asthma and chronic obstructive pulmonary disease. Aliment Pharmacol Ther. 2018;47(2):176-91.

6. Saleh CM, Smout AJ, Bredenoord AJ. The diagnosis of gastro-esophageal reflux disease cannot be made with barium esophagograms. Neurogastroenterol Motil. 2015;27(2):195-200.

7. Macharia EW. Comparison of upper gastrointestinal contrast studies and pH/impedance tests for the diagnosis of childhood gastro-oesophageal reflux. Pediatr Radiol. 2012;42(8):946-51.

8. Yang J, Codreanu I, Servaes S, Zhuang H. Radionuclide Salivagram and Gastroesophageal Reflux Scintigraphy in Pediatric Patients: Targeting Different Types of Pulmonary Aspiration. Clin Nucl Med. 2015;40(7):55963.

9. Woodland P, Singendonk MMJ, Ooi J, Nikaki K, Wong
T, Lee C, et al. Measurement of Salivary Pepsin to Detect Gastroesophageal Reflux Disease Is Not Ready for Clinical Application. Clin Gastroenterol Hepatol. 2019;17(3):563-5.

10. Kahrilas PJ, Kia L. Pepsin: A Silent Biomarker for Reflux Aspiration or an Active Player in Extra-Esophageal Mucosal Injury? Chest. 2015;148(2):300-1.

11. Kelly EA, Parakininkas DE, Werlin SL, Southern JF, Johnston N, Kerschner JE. Prevalence of pediatric aspiration-associated extraesophageal reflux disease. JAMA Otolaryngol Head Neck Surg. 2013;139(10):9961001.

12. Doshi A, Bernard-Stover L, Kuelbs C, Castillo E, Stucky E. Apparent Life-Threatening Event Admisions and Gastroesophageal Reflux Disease. Pediatr Emer Care. 2012;28:17-21.

13. Singendonk MMJ, Jaime F, Salvatore S, Tabbers MM, Benninga MA, Vandenplas Y. Multichannel Intraluminal Impedance and $\mathrm{pH}$ Monitoring (pH-MII) in Infants and Children. Gastroesophageal Reflux in Children2017. p. 89-110.

14. Hicks DM, Ours TM, Abelson TI, Vaezi MF, Richter JE. The Prevalence of Hypopharynx Findings Associated with Gastroesophageal Reflux in Normal Volunteers. Journal of Voice. 2002;16(4):564-79.

15. Belafsky P, Postma N, Koufman J. The Validity and Reliability of the Reflux Finding Score (RFS). The Laryngoscope. 2001;111:1313-7.

16. Rosen R, Mitchell PD, Amirault J, Amin M, Watters K, Rahbar R. The Edematous and Erythematous Airway Does Not Denote Pathologic Gastroesophageal Reflux. J Pediatr. 2017;183:127-31.

17. Zerbib F, Dulery C. Facts and Fantasies on Extraesophageal Reflux: A Gastroenterologist Perspective. J Clin Gastroenterol. 2017;51(9):769-76

18. De Bruyne $P$, Ito $S$. Toxicity of long-term use of proton pump inhibitors in children. Arch Dis Child. 2018;103(1):78-82.

19. Blondeau K, Mertens V, Dupont L, Pauwels A, Farre $R$, Malfroot $A$, et al. The relationship between gastroesophageal reflux and cough in children with chronic unexplained cough using combined impedancepH-manometry recordings. Pediatr Pulmonol. 2011;46(3):286-94.

20. Chang AB, Oppenheimer JJ, Kahrilas PJ, Kantar A, Rubin BK, Weinberger $\mathrm{M}$, et al. Chronic Cough and Gastroesophageal Reflux in Children: CHEST Guideline and Expert Panel Report. Chest. 2019;156(1):131-40.

21. Blake K, Teague WG. Gastroesophageal reflux disease and childhood asthma. Curr Opin Pulm Med. 2013;19(1):24-9.

22. Ambartsumyan L, Nurko S, Rosen R. Gastrointestinal Dysmotility and the Implications for Respiratory Disease. Curr Treat Options Pediatr. 2019;5(2):197-214.

23. Davidson G, Wenzl TG, Thomson M, Omari T, Barker $P$, Lundborg $P$, et al. Efficacy and safety of once-daily esomeprazole for the treatment of gastroesophageal reflux disease in neonatal patients. $J$ Pediatr. 2013;163(3):692-8 e1-2. 